Review Article

\title{
Liver involvement in Gaucher disease: A practical review for the hepatologist and the gastroenterologist
}

\author{
Francesca Carubbi ${ }^{\mathrm{a}, *}$, Maria Domenica Cappellini ${ }^{\mathrm{b}, \mathrm{c}}$, Silvia Fargion ${ }^{\mathrm{d}}$, \\ Anna Ludovica Fracanzani ${ }^{\mathrm{d}, \mathrm{e}}$, Fabio Nascimbeni ${ }^{\mathrm{a}}$ \\ ${ }^{a}$ Regional Referral Centre for Lysosomal Storage Diseases, Division of Internal Medicine and Metabolism, Civil Hospital, AOU of Modena, University of \\ Modena and Reggio Emilia, Modena, Italy \\ b Rare Diseases Center, Department of Medicine, “Ca' Granda" Foundation IRCCS, Policlinico Hospital, Milan, Italy \\ ${ }^{\mathrm{c}}$ Department of Clinical Sciences and Community Health, University of Milan, Milan, Italy \\ d “Ca' Granda" Foundation IRCCS, Policlinico Hospital, University of Milan, Milan, Italy \\ e Department of Pathophysiology and Transplantation, Unit of Medicine and Metabolic Disorders, Milan, Italy
}

\section{A R T I C L E I N F O}

\section{Article history:}

Received 27 October 2019

Accepted 12 January 2020

Available online $\mathrm{xxx}$

\section{Keywords:}

Diagnosis

Glucocerebrosidase deficiency

Liver disease

Splenomegaly

\begin{abstract}
A B S T R A C T
Gaucher disease (GD), a rare lysosomal storage disorder caused by deficient glucocerebrosidase activity and consequent accumulation of glycosphingolipids in the mononuclear phagocyte system, may progress to disabling and potentially life-threatening complications when left undiagnosed and untreated. Unfortunately, because of non-specific signs and symptoms and lack of awareness, patients with type $1 \mathrm{GD}$, the most common non-neuropathic variant, frequently experience diagnostic delays. Since splenomegaly and thrombocytopenia are the dominant clinical features in many GD patients leading to first medical contact, the hepatologist and the gastroenterologist need to be aware of this condition. Liver involvement has been reported in the majority of GD patients, and comprises hepatomegaly, with or without liver enzymes alteration, fibrosis/cirrhosis, portal hypertension, focal liver lesions, and cholelithiasis. Moreover, GD is associated with several biochemical alterations of potential interest for the hepatologist and the gastroenterologist, including hypergammaglobulinemia, hyperferritinemia and metabolic abnormalities, that may lead to misdiagnoses with chronic liver diseases of common etiology, such as primary hemochromatosis, autoimmune liver diseases or nonalcoholic fatty liver disease. This comprehensive review, based on the collaborative experience of physicians managing patients with GD, provides practical information on the clinical, histological and radiological hepatic manifestations of GD aiming at facilitating the diagnosis of GD for the hepatologist and the gastroenterologist.
\end{abstract}

(c) 2020 Editrice Gastroenterologica Italiana S.r.l. Published by Elsevier Ltd. All rights reserved.

\section{Introduction}

Gaucher disease (GD) is a rare autosomal recessive disease with an estimated incidence of 1:22.000 newborns due to deficient lysosomal glucocerebrosidase (GBA) enzyme activity leading to glycosphingolipid storage in cells of the mononuclear phagocyte system [1]. It is a chronic progressive multisystem disorder, whose clinical spectrum encompasses hepato-splenomegaly, bone marrow infiltration with thrombocytopenia and anemia, bleeding diathesis, bone disease ranging from osteopenia/osteoporosis to bone pain/bone crisis, lytic lesions/fractures, osteonecrosis and skeletal deformities, and variable central nervous system

\footnotetext{
* Corresponding author at: Regional Referral Centre for Lysosomal Storage Diseases, Division of Internal Medicine and Metabolism, Civil Hospital, AOU of Modena, University of Modena and Reggio Emilia, Via Giardini 1355, 41126, Modena, Italy.

E-mail address: carubbi@unimore.it (F. Carubbi).
}

involvement [2-5]. GD is conventionally categorized in three main phenotypes on the ground of neurologic involvement: type $1 \mathrm{GD}$, the most common, frequently adult-onset, non-neuropathic variant; type $2 \mathrm{GD}$, the lethal infantile, acute neuropathic form; and type $3 \mathrm{GD}$, the severe chronic neuro-visceral form [2,4]. However, this classification is somewhat artificial, since GD actually manifests as a clinical continuum; indeed, it is now clear that also type $1 \mathrm{GD}$ may be associated with neurologic manifestations, in particular movement disorders and Parkinson disease $[4,6]$. Few genotype-phenotype correlations have been identified. The most recognized correlation concerns patients who carry the common N370S mutation on at least one allele of the GBA gene. N370S mutation is encountered only in subjects with type $1 \mathrm{GD}$ and is protective for the development of the neurological involvement characteristic of type 2 and type 3 GD. Subjects who are homozygous for the N370S mutation can also remain poorly symptomatic until adulthood and can escape medical attention [7]. Other clinical features of GD are: immunological alterations, 
including polyclonal and monoclonal gammopathy, high prevalence of autoantibodies and increased risk of malignancies, notably including multiple myeloma and hepatocellular carcinoma (HCC) [8-10]. Metabolic abnormalities, due to chronic inflammation and characterized by hypercatabolic state with growth retardation, chronic fatigue and reduced body mass, dyslipidemia with low high-density lipoprotein (HDL) cholesterol and hyperferritinemia without significant iron overload could also be present $[11,12]$. Finally, cardio-pulmonary involvement, with interstitial lung disease, pulmonary hypertension and valvulopathies, has also been described in most severe cases [13,14].

Since hepato-splenomegaly and thrombocytopenia are the dominant clinical features in many GD patients leading to first medical contact, the hepatologist and the gastroenterologist need to be aware of this condition in their routine practice. Moreover, misdiagnoses with other chronic liver diseases, such as primary hemochromatosis, autoimmune liver diseases or nonalcoholic fatty liver disease (NAFLD), may be possible due to the abovementioned association of GD with hyperferritinemia, hypergammaglobulinemia and metabolic abnormalities [15,16].

Surveys of patients and medical specialists have clearly shown that, because of non-specific symptoms and lack of awareness, prolonged diagnostic delays are very common in GD and may result in severe disease complications, disabilities and potentially life-threatening manifestations $[5,17,18]$. Physician education may increase the likelihood of prompt detection of GD and allow its correct management with specific treatment, such as enzyme replacement therapy (ERT) or substrate reduction therapy (SRT), that are effective in preventing or reversing GD-related complications. Three recombinant glucocerebrosidase preparations, imiglucerase, velaglucerase alfa and taliglucerase alfa, are currently available. These ERT, which are administered i.v. once every two weeks, have been shown to improve hematological, visceral and skeletal manifestations of GD, and the quality of life of affected patients. The SRT eliglustat, based on the inhibition of glucosylceramide synthase, is now an alternative oral first-line therapeutic option, whose efficacy is comparable to that of ERT [19,20]. Screening strategies for GD by using simple diagnostic algorithms in selected high-risk populations, such as pediatric or adult subjects with splenomegaly and/or thrombocytopenia, have proven feasible and effective in helping pediatricians and hematologists in promoting a timely diagnosis $[21,22]$. Of note, due to the high GD prevalence among Ashkenazi Jews, Mistry et al. have even proposed to screen for GD as a first-line investigation in Ashkenazi patients presenting with splenomegaly [2].

This comprehensive review, based on the collaborative experience of physicians managing patients with GD and chronic liver diseases of various etiology, provides practical information on the clinical, histological and radiological hepatic manifestations of GD aiming at facilitating the diagnosis of GD for the hepatologist and the gastroenterologist.

\section{Hepatic manifestations of Gaucher disease}

A certain degree of liver involvement with a wide variability in clinical presentation has been reported in the majority of GD patients [23]. Hepatic manifestations of GD comprise hepatomegaly, with or without liver enzymes alteration, fibrosis, cirrhosis and portal hypertension, HCC and non-HCC focal liver lesions and cholelithiasis. Moreover, GD is associated with several biochemical abnormalities that may be of potential interest for the hepatologists/gastroenterologists, such as hypergammaglobulinemia and hyperferritinemia. Finally, concurrent chronic liver diseases, such as liver steatosis, chronic viral hepatitis and vascular liver diseases may be frequently found in GD patients, making the diagnosis of GD more difficult.

\subsection{Hepatomegaly and liver enzymes abnormalities}

Liver volume, measured by imaging techniques, is conventionally reported in multiples of normal size predicted for body weight, which is $2.5 \%$ of total body weight in kilograms [24]. Moderate hepatomegaly is defined as a liver volume $>1.25$ and $\leq 2.5$ multiples of normal and severe hepatomegaly as a liver volume $>2.5$ multiples of normal [25]. Some degree of liver enlargement is found in nearly all GD patients as a consequence of intra-hepatic accumulation of Gaucher cells and secondary inflammatory response. However, since liver enlargement is usually less severe than spleen enlargement, if hepatomegaly outweighs splenomegaly, then a careful evaluation for other causes of liver disease is necessary. Data from the International Collaborative Gaucher Group Registry show that moderate or severe hepatomegaly is present in at least $80 \%$ of patients with type $1 \mathrm{GD}$ at the time of first infusion of ERT; of note, in non-splenectomized subjects mean liver volume is 1.8 multiples of normal whereas mean spleen volume is 19.4 multiples of normal [25]. Liver volume is higher in patients with more severe GD and in splenectomized patients. Therapy for GD, both ERT and SRT, is effective in reducing or normalizing liver volume; however, hepatomegaly may persist despite treatment when severe and nodular hepatomegaly, significant liver fibrosis or splenectomy are present $[19,20,25,26]$.

Liver enzymes, particularly aminotransferases and alkaline phosphatase, may be elevated in GD patients. However, the increase in liver enzymes is usually mild or moderate, and does not correlate with hepatomegaly or severity of liver involvement [27].

\subsection{Liver histology in Gaucher disease}

Only few studies have provided detailed histologic descriptions of liver abnormalities in GD. James et al., who described the liver histopathological findings of 22 untreated GD patients, showed a wide range of pathologic changes spanning from the presence of scattered foci of storage cells with little structural abnormalities of the liver to cirrhosis with extensive replacement of the liver by storage cells [28]. Storage cells, defined as Gaucher cells, are sphingolipid-laden macrophages displaying small round vesicular nuclei and abundant lightly eosinophilic cytoplasm with a wrinkled or striated appearance, and may virtually be found in every liver biopsy of GD patients. In the majority of GD patients, Gaucher cells have a zonal distribution, with central zones (zone 3) showing the highest abundance, portal and periportal regions (zone 1) showing mild to moderate presence, and midzones presenting only occasional scattered foci of storage cells [28]. Gaucher cells often present a slight accumulation of iron. Hepatocytes, which do not accumulate the storage material, may present degenerative changes and atrophy when are adjacent to or within large collections of Gaucher cells. Inflammatory changes are mild to moderate and are closely associated with Gaucher cells. At least pericellular liver fibrosis is present in nearly all GD patients, and a significant proportion may develop severe fibrosis with thick fibrous septa, predominantly originating from the central zones where the storage of Gaucher cells is more represented, or cirrhosis [28]. The severity of histologic liver involvement is associated with the presence of severe extra-hepatic complications of GD and splenectomy [28].

\subsection{Liver fibrosis and cirrhosis}

Liver fibrosis is a serious complication of GD, since its severity is the main driver for the development of long-term liver-related events, such as cirrhosis, portal hypertension and HCC [10,29]. Although cirrhosis has been only anecdotally reported, significant liver fibrosis is largely underestimated and seems to be common 
in GD patients. In past years, the systematic evaluation of liver fibrosis by liver biopsy in GD patients has been hampered by the fear of an increased bleeding tendency. With the advent of imaging techniques, such as ultrasound (US)-based elastography or magnetic resonance (MR) elastography, which allow a non-invasive and accurate evaluation of liver fibrosis, recent studies were able to provide some estimate of the prevalence of clinically relevant liver fibrosis in GD, ranging from $20 \%$ to $50 \%$ [29-31]. These studies found that splenectomy, the severity of extrahepatic manifestations of GD, and non-N370S GBA genotypes (i.e. genotypes commonly associated with more severe disease), were all associated with the presence and severity of liver fibrosis [29-31]. The severe extreme of the spectrum of GD-related liver involvement has been described by Lachmann et al. in their case series of 4 splenectomized patients with severe multi-organ involvement [32]. All these patients had portal hypertension, decompensated liver failure and severe hepatic parenchymal disease histologically and radiologically characterized by massive confluent fibrosis with focal calcifications occupying the central region of the liver [32]. The mechanism of liver fibrosis in GD may be multifactorial. In the early steps of fibrosis development, the accumulation of Gaucher cells in the liver, which is more marked in severe GD and may be worsened by splenectomy, may act through local release of cytotoxic, proinflammatory and fibrogenic factors. Subsequently, once dense fibrous bands have developed, episodes of ischemia and infarction may play a part in the pathophysiology of liver fibrosis progression, as substantiated by the finding of calcifications within areas of massive fibrosis $[28,32]$. Of note, one study found that the length of ERT is inversely correlated with liver fibrosis [29], and another study showed that, even when fibrosis is advanced, the institution of ERT can reduce the numbers of Gaucher cells infiltrating the liver parenchyma, ameliorate the severity of portal hypertension and prevent variceal bleeding and hepatic decompensation [32], suggesting a beneficial effect of ERT on progression of GD-related liver disease.

\subsection{Portal hypertension}

Portal hypertension may be a rare complication of severe cases of GD and may become clinically significant with development of esophageal varices and occurrence of life-threatening bleeding [32]. Of note, gastrointestinal bleeding and liver failure were among the leading causes of premature death of GD patients in the preERT era, but nowadays are increasingly rare with earlier diagnosis and timely institution of appropriate GD treatment $[33,34]$. Portal hypertension in GD is not just secondary to the presence of liver cirrhosis, since the overflow in the portal system secondary to splenomegaly or the massive infiltration of Gaucher cells in liver parenchyma especially in splenectomized patients have also been reported as potential underlying mechanisms [23]. Even if splenectomy was performed in the past as a therapeutic option for massive splenomegaly with life-threatening pre-hepatic portal hypertension, now this procedure should be discouraged since it may lead to worsening of liver and bone disease and systemic GD complications. Here it is worth acknowledging that splenomegaly and cytopenia in GD are not necessarily associated with chronic liver disease and portal hypertension, rather they are the consequence of the accumulation of Gaucher cells and chronic inflammatory infiltrates in the spleen and bone marrow. As such, splenomegaly and thrombocytopenia are almost invariably present in GD patients independently of the presence of liver disease and portal hypertension.

\subsection{HCC and non-HCC focal liver lesions}

Focal splenic and hepatic lesions are common in GD patients and are closely associated with GD severity. The reported preva- lence of splenic lesions in several GD cohorts ranges from $18 \%$ to $33 \%$; the vast majority of focal splenic abnormalities is represented by Gaucheromas, benign clusters of Gaucher cells associated with areas of fibrosis and iron accumulation [35,36]. The prevalence of hepatic lesions is reported to be slightly lower ranging from $6 \%$ to $25 \%$; the differential diagnosis of focal liver abnormalities is much more complex. Indeed, although focal liver lesions are mostly Gaucheromas, they may represent a diagnostic challenge due to the variable radiological features, which can mimic malignant liver lesions, in particular HCC $[35,37]$. Of note, GD is associated with an increased risk of malignancies, including HCC, as a consequence of immune dysregulation and chronic macrophage activation with increased release of cytokines and cellular dysfunction due to accumulation of glycosphingolipids [38,39]. It has been shown that GD patients with previous splenectomy, advanced fibrosis/cirrhosis, iron overload and concurrent causes of chronic liver disease (i.e. alcohol, viral hepatitis) are those at the highest risk of HCC occurrence and those who may benefit more from surveillance strategies [10,37].

\subsection{Cholelithiasis}

Patients with GD have been found to have a 5-fold excess risk of gallstones as compared to the general population [40]; the prevalence of cholelithiasis in several GD cohorts ranges from 25\% to $46 \%$ and is associated with age, female sex, previous splenectomy and GD severity [40-43]. Of note, bile lipid analyses in GD patients with cholelithiasis has revealed that gallstones are composed predominantly of cholesterol, whereas pigment stones are an exception; moreover, bile lipid composition in GD patients is abnormal and contains glucosylceramide [40].

\subsection{Other GD-related alterations of potential interest for the hepatologists/gastroenterologists}

\subsubsection{Hypergammaglobulinemia and autoantibodies}

The presence of polyclonal gammopathy and autoantibodies is quite common in GD patients, independently of GD severity and splenectomy. The reported prevalence of hypergammaglobulinemia ranges from $14 \%$ to $64 \%$ [44,45]; similarly, the prevalence of autoantibodies has been reported as much as 60\% [46,47]. Importantly, the presence of autoantibodies is not associated with an increased prevalence of clinically manifested autoimmune diseases [47].

\subsubsection{Hyperferritinemia}

Hyperferritinemia is very common in GD patients, affecting up to $87 \%$ of untreated subjects. Ferritin levels are correlated with GD severity and splenectomy and significantly decrease during GD treatment [48-50]. Hyperferritinemia is not associated with systemic iron overload in GD, unless genetic (HFE mutations) or acquired/environmental (splenectomy, alcohol, metabolic syndrome, chronic viral hepatitis, malignancies) concurrent causes of iron storage are present $[30,48]$. The mechanisms underlying hyperferritinemia, which are not yet completely defined, include chronic low-grade inflammation, impaired macrophages functions and local dysregulation in hepcidin-ferroportin axis [51].

\subsubsection{Concurrent liver diseases}

The presence of concurrent liver diseases in GD patients is common. Chronic viral hepatitis and vascular liver diseases are frequent due to splenectomy, often required in the past for the management of severe splenomegaly, other surgical procedures, including gallbladder removal, and blood transfusions for cytopenia [23,29]. Historically, transfusional iron overload has been an important 
cofactor for liver disease progression in GD patients. Liver steatosis is also a prevalent finding in GD patients due to metabolic abnormalities, such as insulin resistance and increased adiposity, associated with GD itself and unhealthy lifestyle habits [11,23]. Moreover, long-term treatment with ERT has been associated with a significant weight gain [11,52].

\section{Imaging characteristics of liver involvement in Gaucher disease}

As detailed above, liver involvement in GD exhibits a wide clinical spectrum with variable severity and an unpredictable natural course. Furthermore, apart from the primary involvement of the liver due to GD, patients may also suffer from other comorbidities involving the liver, thus making the diagnosis and/or the assessment more difficult. Differently from the past, in which US, computed tomography (CT) and/or MR imaging techniques were mainly used to evaluate the increase in organ volume [24], currently the indications to perform imaging techniques in the assessment of liver involvement in GD are: 1 ) the identification of early tissue damage and the evaluation of liver disease severity; 2) the differentiation of Gaucheromas from other benign or malignant focal liver lesions; 3 ) monitoring the response to ERT or SRT.

\subsection{Identification of early tissue damage and evaluation of the severity of liver disease}

US-based methods are the cheapest and most widely available imaging techniques proposed to non-invasively evaluate the degree of tissue damage in GD. US can recognize diffuse irregular liver texture, expression of chronic liver damage, as well as hepatic steatosis. The latter can be frequently detected in GD patients on long-term ERT and with concurrent metabolic syndrome and is probably related to the concomitant presence of NAFLD, rather than being the consequence of glycosphingolipid storage in macrophages [11,29,52]. Doppler US may help in evaluating the severity of liver disease and portal hypertension. Promising results are provided by US-based elastography techniques, transient elastography with Fibroscan ${ }^{\circledR}$ being the most popular one. Several studies have demonstrated the feasibility of Fibroscan ${ }^{\circledR}$ for the evaluation of liver stiffness in GD patients and have suggested that significant fibrosis is an overlooked complication of GD and is strongly associated with GD severity [29-31]. Of note, an interesting study showed that combined liver and spleen elastography, using either Fibroscan ${ }^{\circledR}$ or 2-dimensional share wave elastography, is a useful tool for differentiating GD patients from cirrhotic patients of different etiology among subjects with splenomegaly [31].

MR imaging techniques outweigh the performance of US methods in identifying early tissue damage and evaluating the severity of liver disease. Diffusion-weighted and chemical shift MR imaging have been proposed for the detection and quantification of hepatic and splenic infiltration in GD and have been associated with GD severity [53,54]. Some MR imaging protocols, such as T2* and relaxation rate $\mathrm{R} 2 *$ measurements, or the Gandon methods, are able to provide reliable estimates of liver iron concentrations. Bohte et al. found an association between liver iron concentration measured through MR and splenectomy in GD patients [30]. The use of wholebody MR R2* measurements has also been proposed in GD patients; a study showed that the presence of increased $\mathrm{R}^{*}$ values in liver, bone marrow and splenic Gaucheromas was quite common in GD, was associated with ferritin levels and presumably indicated elevated tissue iron concentrations [55]. Liver stiffness measurement by MR elastography is considered a reliable non-invasive tool to quantify liver fibrosis in patients with chronic viral hepatitis or NAFLD [56-58]; however, the use of these MR techniques in clin- ical practice is still limited and data on GD patients are scanty. In a small cohort of adult patients with GD, liver stiffness values, as measured by both Fibroscan ${ }^{\circledR}$ and MR elastography, were associated with GD severity and splenectomy [30]. A larger recent study confirmed that MR elastography may be a useful tool for monitoring disease severity and progression by showing a positive correlation with GD severity scoring system GD-DS3 [59].

\subsection{Differentiation of Gaucheromas from other benign or malignant focal liver lesions}

The prevalence of focal liver lesions has been reported to range from $6 \%$ to $25 \%[35,60]$, and is more frequent in patients with more severe GD [35]. Further to the classic benign (hemangiomas, cysts, focal nodular hyperplasia) and malignant focal liver lesions (HCC), single or multiple nodules in GD patients may be the expression of clusters of Gaucher cells conglomerated in the liver (Gaucheromas). Regenboog et al. [35] reported the imaging characteristics of all focal lesions in liver and spleen in the Dutch GD cohort. Of 95 type 1 GD patients, $40 \%$ had focal splenic and/or hepatic lesions (24\% had splenic lesions, 25\% had liver lesions and 9\% patients had both). Liver lesions identified as Gaucheromas had variable imaging characteristics: hyper- or hypoechoic with calcifications on US, hyper- to hypo-intense on MR, and hypodense on CT usually without contrast enhancement [35]. These non-specific radiological features make difficult to distinguish Gaucheromas from hemangiomas, focal nodular hyperplasia or HCC by conventional US, CT or MR. Dynamic contrast-enhanced studies and, in case of inconclusive results, percutaneous imaging-guided biopsy, are necessary to further characterize indeterminate focal liver lesions. Given the reported increased risk of HCC development in GD [10], US is widely recommended for regular routine follow-up in high-risk patients (i.e. those with liver cirrhosis, concurrent chronic liver diseases, and/or splenectomy).

\subsection{Monitoring the response to enzyme replacement therapy or substrate reduction therapy}

ERT and SRT are very effective in reducing hepatic and splenic volumes within $1-2$ years $[20,25]$. Imaging techniques that evaluate liver and spleen volumes are used for monitoring disease activity and facilitating treatment decision-making. Although US is not very accurate in evaluating organ volumes, a good correlation between liver volume and hepatic longitudinal diameter evaluated by US has been found in patients with GD, making this method useful for routine monitoring when MR imaging techniques are not available $[23,61]$. CT is not suitable for regular follow-up of GD patients since it is associated with exposure to ionizing radiation. Despite its high costs, MR is considered the gold standard technique in assessing therapeutic efficacy since it allows to have, at the same time, information on the reduction of organ volumes and on the evolution of bone disease by evaluating the severity of bone marrow infiltration and/or the presence of bone lesions or osteonecrosis [62].

\section{Diagnostic hints of GD for the hepatologist and the gastroenterologist}

Subjects with hepatomegaly or splenomegaly often consult the hepatologist and/or the gastroenterologist for the suspicion of chronic liver disease. Splenomegaly with or without hepatomegaly is the most common feature of GD; however, this lysosomal disorder is rarely considered as a differential diagnosis in patients with unexplained splenomegaly. To facilitate the potential diagnosis of GD in adult patients presenting to the hepatologist and the gastroenterologist with splenomegaly (or previous splenectomy), here 
Table 1

Diagnostic hints of Gaucher disease for the hepatologist and the gastroenterologist.

Unexplained splenomegaly (or previous splenectomy) plus one or more of

these factors may increase clinical suspicion for Gaucher disease:

Thrombocytopenia

Anemia

Bleeding diathesis

History of growth retardation/chronic fatigue

Hepatomegaly

Spleen nodules/lesions

Gallstones

Low HDL cholesterol

Increased ferritin levels

Policlonal gammopathy

Monoclonal gammopathy, including MGUS

Bone involvement

Parkinsonism

Family history of Parkinson disease

HDL: High-density lipoprotein; MGUS: Monoclonal gammopathy of unknown significance.

Includes: Reduction of bone mineral density, chronic bone pain, acute bone crisis, pathologic fractures, lytic lesions, osteonecrosis, Erlenmeyer flask deformity.

we provide several hints based on the presence/absence of several peculiar signs or symptoms of GD, which must be evaluated when more common causes of splenomegaly have been excluded (Table 1).

When GD is suspected, the first step in the diagnostic work-up is the detection of a reduced GBA activity that can be evaluated on dried blood spots (DBS). DBS assay is a quick, not expensive and accurate screening test; it can be easily performed in any outpatient service and it has been validated in a large number of patients [63]. A pathologic result on DBS should be confirmed by evaluating GBA enzymatic activity in blood leukocytes or cell cultures and DNA sequencing with evidence of biallelic GBA pathogenic mutations.

\section{Conclusions and future perspectives}

GD is a chronic and multisystem disease which may progress to severe disabling and potentially life-threatening complications when left undiagnosed and untreated $[17,18]$. Several diagnostic algorithms for GD have previously been proposed for hematologists and pediatricians, with the aims of increasing awareness and favoring an early diagnosis [21,22]. Liver involvement in GD is nonspecific and variable [11,23]; as a consequence, GD is frequently misdiagnosed with other highly prevalent chronic liver diseases of different etiologies [15,16]. Splenomegaly with or without hepatomegaly is a nearly universal finding; as such, GD should be considered as a differential diagnosis in patients with unexplained splenomegaly referred to the hepatologist or the gastroenterologist. Other peculiar findings that should increase the suspicion of GD are the presence of bone involvement, gammopathy, hyperferritinemia, hepatomegaly, hepatic and splenic nodules, gallstones, low HDL cholesterol, thrombocytopenia, anemia, bleeding diathesis, chronic fatigue, familial or personal history of parkinsonism $[2,4,5]$. In this paper, we have provided a comprehensive and practical review of liver involvement in GD aimed at facilitating a prompt diagnosis of GD for the hepatologist and the gastroenterologist.

\section{Conflict of interest}

All authors are members of Sanofi-Genzyme advisory boards.

\section{Acknowledgement}

Authors would like to thank Doctor Luigi Maria De Matteis for medical writing support.

\section{References}

[1] Burlina AB, Polo G, Salviati L, Duro G, Zizzo C, Dardis A, et al. Newborn screening for lysosomal storage disorders by tandem mass spectrometry in North East Italy. J Inherit Metab Dis 2018;41:209-19.

[2] Mistry PK, Cappellini MD, Lukina E, Ozsan H, Mach Pascual S, Rosenbaum H, et al. A reappraisal of Gaucher disease-diagnosis and disease management algorithms. Am J Hematol 2011;86:110-5.

[3] Hughes D, Mikosch P, Belmatoug N, Carubbi F, Cox T, Goker-Alpan O, et al. Gaucher disease in bone: from pathophysiology to practice. J Bone Miner Res 2019;34:996-1013.

[4] Mistry PK, Lopez G, Schiffmann R, Barton NW, Weinreb NJ, Sidransky E. Gaucher disease: progress and ongoing challenges. Mol Genet Metab 2017:120:8-21.

[5] Mehta A, Kuter DJ, Salek SS, Belmatoug N, Bembi B, Bright J, et al. Presenting signs and patient co-variables in Gaucher disease: outcome of the Gaucher Earlier Diagnosis Consensus (GED-C) Delphi initiative. Intern Med J 2019:49:578-91.

[6] Aharon-Peretz J, Rosenbaum H, Gershoni-Baruch R. Mutations in the glucocerebrosidase gene and Parkinson's disease in Ashkenazi Jews. N Engl J Med 2004:351:1972-7.

[7] Hruska KS, LaMarca ME, Scott CR, Sidransky E. Gaucher disease: mutation and polymorphism spectrum in the glucocerebrosidase gene (GBA). Human Mutat 2008;29:567-83.

[8] Arends M, van Dussen L, Biegstraaten M, Hollak CE. Malignancies and monoclonal gammopathy in Gaucher disease; a systematic review of the literature. Br J Haematol 2013;161:832-42.

[9] Nair S, Branagan AR, Liu J, Boddupalli CS, Mistry PK, Dhodapkar MV. Clonal immunoglobulin against lysolipids in the origin of myeloma. N Engl J Med 2016;374:555-61.

[10] Regenboog M, van Dussen L, Verheij J, Weinreb NJ, Santosa D, Vom Dahl S, et al Hepatocellular carcinoma in Gaucher disease: an international case series. J Inherit Metab Dis 2018;41:819-27.

[11] Nascimbeni F, Dalla Salda A, Carubbi F. Energy balance, glucose and lipid metabolism, cardiovascular risk and liver disease burden in adult patients with type 1 Gaucher disease. Blood Cells Mol Dis 2018;68:74-80.

[12] Regenboog M, van Kuilenburg AB, Verheij J, Swinkels DW, Hollak CE. Hyperferritinemia and iron metabolism in Gaucher disease: potential pathophysiological implications. Blood Rev 2016:30:431-7.

[13] Miller A, Brown LK, Pastores GM, Desnick RJ. Pulmonary involvement in type 1 Gaucher disease: functional and exercise findings in patients with and without clinical interstitial lung disease. Clin Genet 2003:63:368-76.

[14] Mistry PK, Sirrs S, Chan A, Pritzker MR, Duffy TP, Grace ME, et al. Pulmonary hypertension in type 1 Gaucher's disease: genetic and epigenetic determinants of phenotype and response to therapy. Mol Genet Metab 2002;77: 91-8.

[15] Saadi T, Rosenbaum H, Veitsman E, Baruch Y. Gaucher's disease type I: a disease masked by the presence of abnormal laboratory tests common to primary liver disease. Eur J Gastroenterol Hepatol 2010;22:1019-21.

[16] vom Dahl S, Mengel E. Lysosomal storage diseases as differential diagnosis of hepatosplenomegaly. Best Pract Res Clin Gastroenterol 2010;24:619-28.

[17] Mistry PK, Sadan S, Yang R, Yee J, Yang M. Consequences of diagnostic delays in type 1 Gaucher disease: the need for greater awareness among hematologistsoncologists and an opportunity for early diagnosis and intervention. Am J Hematol 2007;82:697-701.

[18] Mehta A, Belmatoug N, Bembi B, Deegan P, Elstein D, Goker-Alpan O, et al. Exploring the patient journey to diagnosis of Gaucher disease from the perspective of 212 patients with Gaucher disease and 16 Gaucher expert physicians. Mol Genet Metab 2017;122:122-9.

[19] Mistry PK, Batista JL, Andersson HC, Balwani M, Burrow TA, Charrow J, et al. Transformation in pretreatment manifestations of Gaucher disease type 1 during two decades of alglucerase/imiglucerase enzyme replacement therapy in the International Collaborative Gaucher Group (ICGG) Gaucher Registry. Am J Hematol 2017;92:929-39.

[20] Lukina E, Watman N, Dragosky M, Lau H, Avila Arreguin E, Rosenbaum H, et al. Outcomes after 8 years of eliglustat therapy for Gaucher disease type 1: final results from the Phase 2 trial. Am J Hematol 2019;94:29-38.

[21] Motta I, Filocamo M, Poggiali E, Stroppiano M, Dragani A, Consonni D, et al. A multicentre observational study for early diagnosis of Gaucher disease in patients with Splenomegaly and/or Thrombocytopenia. Eur J Haematol 2016;96:352-9.

[22] Di Rocco M, Andria G, Deodato F, Giona F, Micalizzi C, Pession A. Early diagnosis of Gaucher disease in pediatric patients: proposal for a diagnostic algorithm. Pediatr Blood Cancer 2014:61:1905-9.

[23] Adar T, Ilan Y, Elstein D, Zimran A. Liver involvement in Gaucher disease review and clinical approach. Blood Cells Mol Dis 2018;68:66-73.

[24] Elstein D. Hadas-Halpern I, Azuri Y, Abrahamov A, Bar-Ziv Y,Zimran A. Accuracy of ultrasonography in assessing spleen and liver size in patients with Gaucher disease: comparison to computed tomographic measurements. J Ultrasound Med 1997; 16:209-11

[25] Weinreb NJ, Goldblatt J, Villalobos J, Charrow J, Cole JA, Kerstenetzky M, et al. Long-term clinical outcomes in type 1 Gaucher disease following 10 years of imiglucerase treatment. J Inherit Metab Dis 2013;36:543-53.

[26] Shemesh E, Deroma L, Bembi B, Deegan P, Hollak C, Weinreb NJ, et al. Enzyme replacement and substrate reduction therapy for Gaucher disease. Cochrane Database Syst Rev 2015:CD010324. 
[27] Zimran A, Kay A, Gelbart T, Garver P, Thurston D, Saven A, et al. Gaucher disease. Clinical, laboratory, radiologic, and genetic features of 53 patients. Medicine 1992;71:337-53.

[28] James SP, Stromeyer FW, Chang C, Barranger JA. Liver abnormalities in patients with Gaucher's disease. Gastroenterology 1981;80:126-33.

[29] Nascimbeni F, Cassinerio E, Dalla Salda A, Motta I, Bursi S, Donatiello S, et al. Prevalence and predictors of liver fibrosis evaluated by vibration controlled transient elastography in type 1 Gaucher disease. Mol Genet Metab 2018;125:64-72.

[30] Bohte AE, van Dussen L, Akkerman EM, Nederveen AJ, Sinkus R, Jansen PL, et al. Liver fibrosis in type I Gaucher disease: magnetic resonance imaging, transient elastography and parameters of iron storage. PLoS One 2013;8:e57507.

[31] Webb M, Zimran A, Dinur T, Shibolet O, Levit S, Steinberg DM, et al. Are transient and shear wave elastography useful tools in Gaucher disease? Blood Cells Mol Dis 2018;68:143-7.

[32] Lachmann RH, Wight DG, Lomas DJ, Fisher NC, Schofield JP, Elias E, et al. Massive hepatic fibrosis in Gaucher's disease: clinico-pathological and radiological features. OJM 2000;93:237-44.

[33] Weinreb NJ, Barbouth DS, Lee RE. Causes of death in 184 patients with type 1 Gaucher disease from the United States who were never treated with enzyme replacement therapy. Blood Cells Mol Dis 2018;68:211-7.

[34] Weinreb NJ, Deegan P, Kacena KA, Mistry P, Pastores GM, Velentgas P, et al. Life expectancy in Gaucher disease type 1. Am J Hematol 2008;83:896-900.

[35] Regenboog M, Bohte AE, Somers I, van Delden OM, Maas M, Hollak CE. Imaging characteristics of focal splenic and hepatic lesions in type 1 Gaucher disease. Blood Cells Mol Dis 2016;60:49-57.

[36] Stein P, Malhotra A, Haims A, Pastores GM, Mistry PK. Focal splenic lesions in type I Gaucher disease are associated with poor platelet and splenic response to macrophage-targeted enzyme replacement therapy. J Inherit Metab Dis 2010;33:769-74.

[37] Starosta RT, Pinto EVF, Dornelles AD, Cerski CTS, Alvares-da-Silva MR, Schwartz IVD. Hepatocellular carcinoma in Gaucher disease: reinforcing the proposed guidelines. Blood Cells Mol Dis 2019;74:34-6.

[38] de Fost M, Vom Dahl S, Weverling GJ, Brill N, Brett S, Haussinger D, et al. Increased incidence of cancer in adult Gaucher disease in Western Europe. Blood Cells Mol Dis 2006;36:53-8.

[39] Weinreb NJ, Lee RE. Causes of death due to hematological and nonhematological cancers in 57 US patients with type 1 Gaucher disease who were never treated with enzyme replacement therapy. Crit Rev Oncog 2013;18:177-95.

[40] Taddei TH, Dziura J, Chen S, Yang R, Hyogo H, Sullards C, et al. High incidence of cholesterol gallstone disease in type 1 Gaucher disease: characterizing the biliary phenotype of type 1 Gaucher disease. J Inheri Metab Dis 2010;33: 291-300.

[41] Ben Harosh-Katz M, Patlas M, Hadas-Halpern I, Zimran A, Elstein D. Increased prevalence of cholelithiasis in Gaucher disease: association with splenectomy but not with gilbert syndrome. J Clin Gastroenterol 2004;38:586-9.

[42] Rosenbaum H, Sidransky E. Cholelithiasis in patients with Gaucher disease. Blood Cells Mol Dis 2002;28:21-7.

[43] Zimmermann A, Popp RA, Al-Khzouz C, Bucerzan S, Nascu I, Leucuta D, et al. Cholelithiasis in patients with Gaucher disease type 1: risk factors and the role of ABCG5/ABCG8 gene variants. J Gastrointestin Liver Dis 2016;25:447-55.

[44] Brautbar A, Elstein D, Pines G, Abrahamov A, Zimran A. Effect of enzyme replacement therapy on gammopathies in Gaucher disease. Blood Cells Mol Dis 2004;32:214-7

[45] de Fost M, Out TA, de Wilde FA, Tjin EP, Pals ST, van Oers MH, et al. Immunoglobulin and free light chain abnormalities in Gaucher disease type I: data from an adult cohort of 63 patients and review of the literature. Ann Hematol 2008;87:439-49

[46] Shoenfeld Y, Beresovski A, Zharhary D, Tomer Y, Swissa M, Sela E, et al. Natural autoantibodies in sera of patients with Gaucher's disease. J Clinical Immunol 1995;15:363-72.

[47] Serratrice C, Bensalah N, Penaranda G, Bardin N, Belmatoug N, Masseau A, et al Prevalence of autoantibodies in the course of Gaucher disease type 1: a multicenter study comparing Gaucher disease patients to healthy subjects. Joint Bone Spine 2018;85:71-7.

[48] Stein P, Yu H, Jain D, Mistry PK. Hyperferritinemia and iron overload in type 1 Gaucher disease. Am J Hematol 2010;85:472-6.

[49] Mekinian A, Stirnemann J, Belmatoug N, Heraoui D, Fantin B, Fain O, et al. Ferritinemia during type 1 Gaucher disease: mechanisms and progression under treatment. Blood Cells Mol Dis 2012;49:53-7.

[50] Lorenz F, Pawlowicz E, Klimkowska M, Beshara S, Bulanda Brustad A, Skotnicki $A B$, et al. Ferritinemia and serum inflammatory cytokines in Swedish adults with Gaucher disease type 1. Blood Cells Mol Dis 2018;68:35-42.

[51] Lefebvre T, Reihani N, Daher R, de Villemeur TB, Belmatoug N, Rose C, et al. Involvement of hepcidin in iron metabolism dysregulation in Gaucher disease. Haematologica 2018;103:587-96.

[52] Langeveld M, de Fost M, Aerts JM, Sauerwein HP, Hollak CE. Overweight, insulin resistance and type II diabetes in type I Gaucher disease patients in relation to enzyme replacement therapy. Blood Cells Mol Dis 2008;40:428-32.

[53] Razek A, Abdalla A, Barakat T, El-Taher H, Ali K. Assessment of the liver and spleen in children with Gaucher disease type I with diffusion-weighted MR imaging. Blood Cells Mol Dis 2018;68:139-42.

[54] Abdel Razek AAK, Barakat T, Ali K. Assessment of liver and spleen in children with caucher disease type 1 with chemical shift imaging. J Comput Assist Tomogr 2019;43:183-6.

[55] Regenboog M, Bohte AE, Akkerman EM, Stoker J, Hollak CEM. Iron storage in liver, bone marrow and splenic Gaucheroma reflects residual disease in type 1 Gaucher disease patients on treatment. Br J Haematol 2017:179:635-47.

[56] Huwart L, Sempoux C, Vicaut E, Salameh N, Annet L, Danse E, et al. Magnetic resonance elastography for the noninvasive staging of liver fibrosis. Gastroenterology 2008;135:32-40.

[57] Yin M, Talwalkar JA, Glaser KJ, Manduca A, Grimm RC, Rossman PJ, et al. Assessment of hepatic fibrosis with magnetic resonance elastography. Clin Gastroenterol Hepatol 2007;1213:e1202.

[58] Singh S, Venkatesh SK, Wang Z, Miller FH, Motosugi U, Low RN, et al. Diagnostic performance of magnetic resonance elastography in staging liver fibrosis: a systematic review and meta-analysis of individual participant data. Clin Gastroenterol Hepatol 2015;451, 440-451 e446.

[59] Serai SD, Naidu AP, Andrew Burrow T, Prada CE, Xanthakos S, Towbin AJ. Correlating liver stiffness with disease severity scoring system (DS3) values in Gaucher disease type 1 (GD1) patients. Mol Genet Metab 2018;123:357-63.

[60] Neudorfer O, Hadas-Halpern I, Elstein D, Abrahamov A, Zimran A. Abdominal ultrasound findings mimicking hematological malignancies in a study of 218 Gaucher patients. Am J Hematol 1997;55:28-34.

[61] Elstein D, Tiomkin M, Hadas-Halpern I, Zimran A. Organ volume by computed tomography correlates with longitudinal axis on ultrasound in patients with Gaucher disease. Ultrasound Q 2011;27:225-8.

[62] Poll LW, Cox ML, Godehardt E, Steinhof V, vom Dahl S. Whole body MRI in type I Gaucher patients: evaluation of skeletal involvement. Blood Cells Mol Dis 2011;46:53-9.

[63] Stroppiano M, Calevo MG, Corsolini F, Cassanello M, Cassinerio E, Lanza F, et al Validity of beta-D-glucosidase activity measured in dried blood samples for detection of potential Gaucher disease patients. Clin Biochem 2014;47:1293-6. 